\title{
General Practice and Medicare Locals, Best Placed to Curb Australia's Chronic Disease Epidemic
}

\section{Abhijeet Ghosh*}

Member, Grand Pacific Health Ltd., Trading as Illawarra-Shoalhaven Medicare Local (ISML), Australia

Keeping in mind the burgeoning chronic disease problem of Australia, Australian governments have chosen to pay focused attention on certain diseases and conditions that contribute significantly to the burden of illness and injury in the Australian community and have called them, The National Health Priority Areas (NHPAs) [1]. Prevalence estimates from Australian Health Survey 2011-12 [2] indicate that of the National Health Priority Areas, the top long-term health conditions experienced in Australia were:

\section{- $\quad$ Arthritis - 3.3 million people (14.8\%)}

- Mental and behavioural conditions - 3.0 million people (13.6\%)

- $\quad$ Asthma - 2.3 million people (10.2\%)

- Heart disease $e^{\star}-1.0$ million people (4.7\%)

*additionally hypertension accounted for 2.3 million people $(10.2 \%)$

These high rates of chronic and preventable diseases along with an ageing population and rising health care costs pose a serious public health challenge for policy makers and health planners of Australia. While national policies and state wide approaches to preventive health and health promotion are definitely required to address the issue of chronic disease; local and smaller catchment surveillance systems are the need of the hour to continuously implement focused strategies targeting these conditions at small community levels and thereby actively evaluating local health strategies so as to regularly monitor the trends of chronic disease across Australia. On these lines the Population Health and Planning stream at the Illawarra-Shoalhaven Medicare Local which is one of the 61 Medicare Locals formed under the Australian Government's National Health Reform Agreement [3], undertook a pilot study to assess the feasibility of implementing a sentinel site surveillance system in regional NSW (New South Wales) to monitor chronic disease prevalence and its associated risk factors. The study (Sentinel Practices Data Sourcing project) was conducted in one of the major General Practices in the Illawarra-Shoalhaven catchment of regional NSW [4].

From the pilot study we found higher than national average estimates for the age-adjusted prevalence of chronic diseases such as hypertension ( $14.3 \%$ for sample $v s .10 .4 \%$, nationally), anxiety disorders (4.4\% vs. $3.8 \%)$ and obesity/overweight (67.1 vs. 63.4\%). Preventive health assessment items were found to be undersubscribed, ranging from $6-20 \%$ in eligible patients. We concluded that the scope of systematic data collected by patient visits to their General Practitioners, routinely entered into desktop software in General Practice, offers a feasible opportunity for a valid and sensitive surveillance system to be adopted in regional Australia and hence can facilitate monitoring of chronic disease prevalence and its associated risk factors. The inclusion of a larger number of sentinel sites that are generalizable to the population being served would provide an accurate and region-specific system for the purposes of population health planning at the primary care level in order to improve the overall health of the community.

\section{References}

1. Australian Institute of Health and Welfare (2012) National health priority areas.

2. ABS (2013) Australian Health Survey, Key Findings, Cat. No. 4364.0.55.003.

3. DoHA (2011) About National Health Reform.

4. Ghosh A, Charlton KE, Girdo L, Batterham MJ, McDonald K (2013) Addressing the deficiencies in the evidence-base for primary practice in regional Australia - Sentinel Practices Data Sourcing (SPDS) project: a pilot study. BMC Family Practice 14: 109
*Corresponding author: Abhijeet Ghosh, Member, Grand Pacific Health Ltd. trading as Illawarra-Shoalhaven Medicare Local (ISML), Australia Tel: (02) 4220 7600; E-mail: abhijeet_ghosh@gmail.com

Received November 22, 2013; Accepted Feburary 24, 2014; Published Feburary 27, 2014

Citation: Ghosh A (2014) General Practice and Medicare Locals, Best Placed to Curb Australia's Chronic Disease Epidemic. Primary Health Care 4: 151 doi:10.4172/2167-1079.1000151

Copyright: ( 2014 Ghosh A. This is an open-access article distributed under the terms of the Creative Commons Attribution License, which permits unrestricted use, distribution, and reproduction in any medium, provided the original author and source are credited. 